\title{
Generator Bidding in Oligopolistic Electricity Markets Using Optimal Control: Fundamentals and Application
}

\author{
Youfei Liu, Student Member, IEEE, and Felix F. Wu, Fellow, IEEE
}

\begin{abstract}
In this paper, optimal control is applied to study generator bidding in an oligopolistic electricity market. The repeated bidding process in (hourly-based) real-time electricity markets is modeled as a dynamic feedback system; an optimal control problem is then formulated to explore individual generator's long-term/multiperiod optimization behavior. Particularly in our formulation, the periodic property of the system demand is considered. Several lemmas are included for concerning system stability. Based on the necessary conditions for optimality from the Pontryagin maximum principle, a sweeping method is proposed, and an optimal state-feedback control rule is then obtained via backward induction. Numerical results suggest that the generator who unilaterally applies optimal control for generation decisions will obtain more profits. A sensitivity analysis is also performed, identifying these market factors that affect the performance of optimal control.
\end{abstract}

Index Terms-Generator bidding, linear periodic system, oligopolistic electricity markets, optimal control, sensitivity analysis.

\section{NOMENCLATURE}

$q_{i}[t] \quad$ Generator $i$ 's generation at time $t$.

$q_{-i}[t] \quad$ Sum of generations from generator $i$ 's rivals at time $t$.

$Q_{-i}[t] \quad$ Vector of generations from all generators except generator $i$ (for generator $i$ who applies the optimal control, $q_{i}[t]$ is the system control variable, $Q_{-i}[t]$ is the vector of system state variables).

$\pi_{i}[t] \quad$ Generator $i$ 's profit/payoff at time $t$.

$p[t] \quad$ Market-clearing price at time $t$.

$D_{d}[t] \quad$ System aggregated demand at time $t$.

$a_{d}[t], b_{d}[t]$ Coefficients of system linear demand function.

$e_{p}[t], f_{p}[t]$ Coefficients of system inverse demand function.

$\beta_{i}[t] \quad$ Factor representing the speed of generator $i$ 's adjustment.

$q_{i c}[t] \quad$ Exogenous variable in generator $i$ 's dynamic model.

$Q_{-i c}[t] \quad$ Vector of exogenous variables of all generators except generator $i$.

$A_{i j}[t] \quad$ Generator $i$ 's state-feedback gain w.r.t. generator $j$ 's output in the dynamic model. For above discrete variables, the corresponding continuous

Manuscript received May 4, 2005; revised January 25, 2006. Paper no. TPWRS-00269-2005.

The authors are with the Department of Electrical and Electronic Engineering, University of Hong Kong, Hong Kong (e-mail: youfei@eee.hku.hk).

Digital Object Identifier 10.1109/TPWRS.2006.876701 variables are given as $q_{i}(t), q_{-i}(t), Q_{-i}(t), \pi_{i}(t)$ $p(t), D_{d}(t), a_{d}(t), b_{d}(t), e_{p}(t), f_{p}(t), A_{i j}(t), q_{i c}(t)$.

$n \quad$ Number of generators in the market.

$T \quad$ Period of periodic demand function.

$N_{T} \quad$ Planning time horizon in long-term optimization.

$C_{i}\left(q_{i}\right) \quad$ Quadratic production cost function of generator $i$.

$a_{i}, b_{i}, c_{i} \quad$ Coefficients of production cost function $C_{i}\left(q_{i}\right)$.

$A_{-1}[t] \quad$ Matrix formed by estimated elements

$A_{k j}[t](k \neq 1 j \neq 1)$.

$B_{-1}[t] \quad$ Vector formed by estimated elements $A_{k 1}[t](k \neq 1)$;.

$Q_{-1 c}[t] \quad$ Vector formed by exogenous variables of generator 1's rivals, $Q_{-1 c}[t]=\left(q_{2 c}[t], q_{3 c}[t], \ldots, q_{\mathrm{nc}}[t]\right)^{T}$.

$J_{i} \quad$ Generator $i$ 's aggregated profit over a planning time horizon.

$\lambda_{i} \quad$ Co-state variable in Hamilton function.

\section{INTRODUCTION}

$\mathbf{P}$ OWER industry has been viewed as a natural monopoly for a long time. Now, the traditionally regulated power industry has undergone a restructuring process to the deregulated one. It is agreed that the aim of power industry deregulation is to establish a competitive electricity market and thus improve the production efficiency. It is also known that the deregulated electricity market is more akin to an oligopoly, because usually, there are a few dominated generators (market suppliers). In such an oligopolistic electricity market, an individual generator has market power and can manipulate market price via its strategic bidding behavior [1]. To maximize its profits when participating in an electricity market, a generator needs to build an optimal bidding strategy. Many optimal algorithms have been applied to address this issue, such as discrete stochastic optimization through a Markov decision process [2], stochastic optimization with a genetic algorithm and Monte Carlo simulation [3], ordinal optimization [4], and Lagrangian relaxation and stochastic dynamic programming [5]. On the other hand, generator bidding can also be modeled as a supplier game. Game-theory-based methods are thus applied to study generators' strategic behavior and analyze the Nash equilibrium in deregulated electricity markets [6]-[8].

As far as we know, most of the above reported works regard the hourly markets as independent, i.e., the optimal bidding strategy is based on generator's short-term/single-period maximization behavior (or the static optimization based on some 
static models). Actually, the system demand has more or less predictable daily variation. Such temporal effect of system demand makes the market dynamic. Therefore, viewing the competition process as a dynamic feedback system may provide a superior model. Dynamic modeling of electricity markets might provide insights about their efficiency and stability that are not available through those static models.

Recently, several papers have studied the dynamics and stability of oligopolistic electricity markets [9]-[11]. Alvarado [9] studies the stability of electricity markets by means of eigenvalues technique. It is shown that if all generators' supply function slopes are positive and all consumers' benefit function slopes are negative, market stability is assured. In [10], the market bidding process is modeled as a closed-loop dynamic system in which previous and current market states are used as feedback signals. Simulation with the proposed model indicates these factors that determine the level of market power exercised. Reference [11] formulates a dynamic Cournot game for generator bidding and solves it using dynamic programming, a type of optimal control. Its study differs in some ways from our study. In [11], the market dynamic model is a linear stochastic price predicator. All market participants are assumed to make long-term optimization, and the derived solution is called a closed "Nash-Cournot' strategy. The focus in [11] is to compare the efficiency and competitiveness of double-sided auctions with one-sided auctions

In this paper, we follow the same idea as in [12] to model the generation competition process as a dynamic feedback system. In our modeling, the market dynamics originate from a market participant's one-step optimization behavior. First, we define a framework for applying optimal control to generation decisions. Later on, we provide a detailed system formulation. A linear periodic dynamic feedback system is proposed to model the generation bidding process in electricity markets, regarding market clearing price (MCP) as system output feedback. For a generator who makes a long-term/multiperiod decision, its generation problem is formulated as an optimal control problem. We include several lemmas concerning system stability. Using a sweeping method, we obtain an optimal state-feedback control rule with backward induction. Numerical results suggest the generator who applies the optimal control will obtain more profits as long as other generators do not. A sensitivity analysis is also carried out to identify market factors that determine the performance of optimal control.

This paper is organized as follows. In Section III, the general idea of optimal control is presented. Section IV gives a detailed system formulation. In Section V, the issues of system stability and the properties of the optimal control system are discussed. The optimal state-feedback control rule is derived using a sweeping method. A numerical simulation and sensitivity analysis are given in Section VI. A discussion is presented in Section VII. Finally, Section VIII concludes the paper.

\section{FRAMEWORK FOR OPTIMAL CONTROL TO GENERATION BIDDING}

\section{A. Bidding Process as a Dynamic Feedback System}

In hourly-based real-time markets for electricity, generators submit their (hourly) generation bids to an independent system

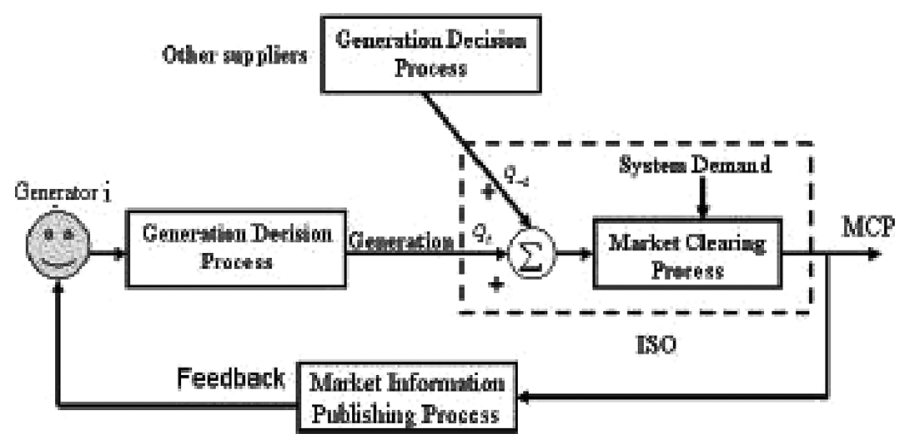

Fig. 1. Diagram of a dynamic feedback system.

operator (ISO). Based on these submitted bids as well as other system information, the ISO clears the market to schedule generation and determine the MCP [13]. After the market is cleared, individual generator knows the publicized MCP and its scheduled generation. In the next round of bidding, the individual generator will adjust its generation bid for maximizing its profits. Therefore, the bidding process can be modeled as a dynamic feedback system, where the MCP is taken as the system output feedback. Fig. 1 shows the general idea of such a dynamic feedback system.

In Fig. 1, there are three function boxes. The generation decision box makes generation bidding decisions; the market clearing box clears the market; and the market information box publishes market clearing results (such as MCP and individual's scheduled generation).

\section{B. Long-Term Optimization With Optimal Control}

As mentioned above, many reported works on generator bidding focus on individual generator's short-term/single-period optimization. That is, the objective function is to maximize the single-period (hourly) profit, i.e. $\max _{q_{i}[t]} \pi_{i}[t]$.

Meanwhile, the long-term/multiperiod optimization intends to maximize the sum of profits over a planning time horizon $N_{T}$, i.e., $\max _{\left\{q_{i}[t]\right\}}\left(\sum_{t=1}^{N_{T}} \pi_{i}[t]\right)$. If the system is always in static or without dynamics, then the hourly profits are independent, and the repetition of single-period optimization is equivalent to the long-term/multiperiod optimization in the sense of aggregated profits, i.e., $\max _{\left\{q_{i}[t]\right\}}\left(\sum_{t=1}^{N_{T}} \pi_{i}[t]\right)=\sum_{t=1}^{N_{T}}\left(\max _{q_{i}[t]} \pi_{i}[t]\right)$. However, hourly profits might not be interdependent, so the long-term/multiperiod optimization could outperform the short-term/single-period optimization, i.e., $\max _{\left\{q_{i}[t]\right\}}\left\{\sum_{t=1}^{N_{T}} \pi_{i}[t]\right\}>\sum_{t=1}^{N_{T}}\left(\max _{q_{i}[t]} \pi_{i}[t]\right)$. In a dynamic system, it is known that multiperiod/long-term optimization can be studied with optimal control. In the following section, we will elaborate on the application of optimal control for generation decisions. Numerical studies are given later to demonstrate the performance of optimal control.

Fig. 2 shows the proposed general idea of applying optimal control to generation decisions. In Fig. 2, the generator (using generator 1 for illustration, for other generators, the idea is similar) will adopt an optimal control strategy for maximizing its aggregated profit over a planning time horizon while taking its observation/estimates of rivals' dynamic behavior as a system state equation.

Note that in the above framework, only one firm's generation is taken as the decision variable. Real electricity markets usu- 


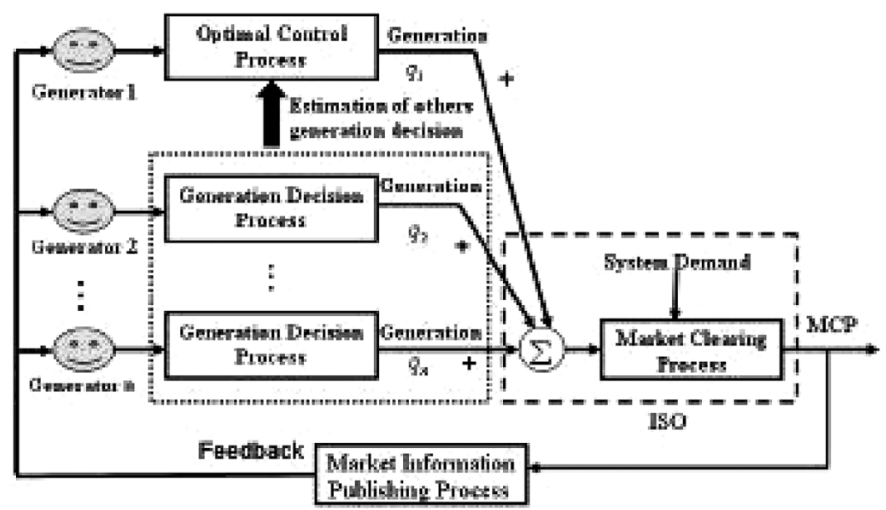

Fig. 2. Applying optimal control to generator bidding.

ally require the generator's bid to be (quantity, price) pairs; thus, the supply function model seems to be the natural choice for studying a generator's strategic bidding [8]. The question is how can our quantity decision process be applicable? In our opinion, a generator's strategic bidding can be thought of as optimally withholding generation capacity to mark up electricity price above the competitive level for more profits. The above quantity decision process thus assumes that an individual generator first determines how much to generate and then bids that specified generation with the corresponding marginal cost. Doing so, the MCP is actually determined with the system demand function (assumed to have price elasticity) and the overall system generation. Usually, the obtained MCP will be larger than a generator's marginal production cost.

\section{SYSTEM FORMULATION}

In this section, a detailed formulation for applying optimal control in a deterministic market situation is given. Market uncertainties are not considered here. This will allow us to analytically solve the problem and tackle the basic issues of solution existence and system stability.

Particularly in our formulation, the periodic property of system demand is considered. We then propose a periodic linear system to model the generator bidding process. With the developed dynamic system, we formulate an optimal control problem to investigate an individual generator's long-term/multiperiod optimization behavior.

\section{A. Linear Periodic System}

Generally, electricity demand can be modeled by some convex function of electricity price. Here we adopt a linear inverse demand function

$$
p(t)=e_{p}(t)-f_{p}(t) D_{d}(t) .
$$

Electricity demand has some price elasticity ]the elasticity is defined as $\left.\xi=\left(\Delta D_{d} / D_{d}\right) \Delta p / p=-(1) /\left(f_{p}\right)(p) /\left(D_{d}\right)\right]$. The elasticity $\xi$ is the consumer's sensitivity to price. Usually, $\xi$ is negative, as demand decreases when price increases. Residential consumers have low price elasticity or have little price response ability, while industrial consumers have higher price elasticity [14].

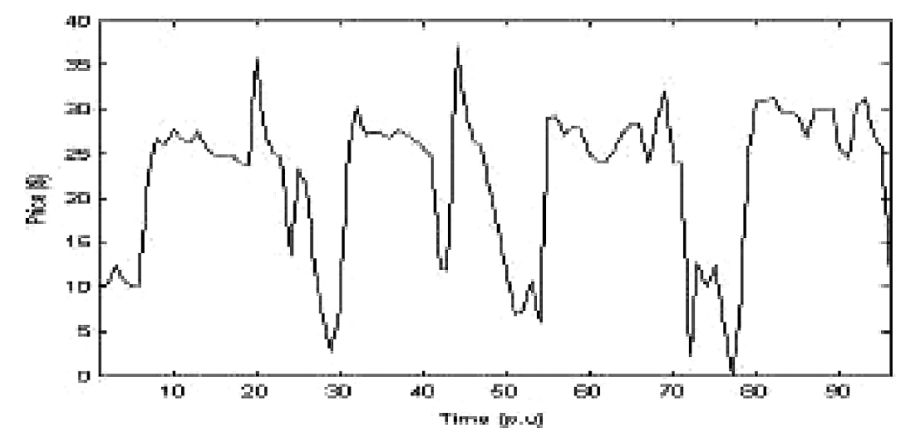

Fig. 3. Unconstrained market price.

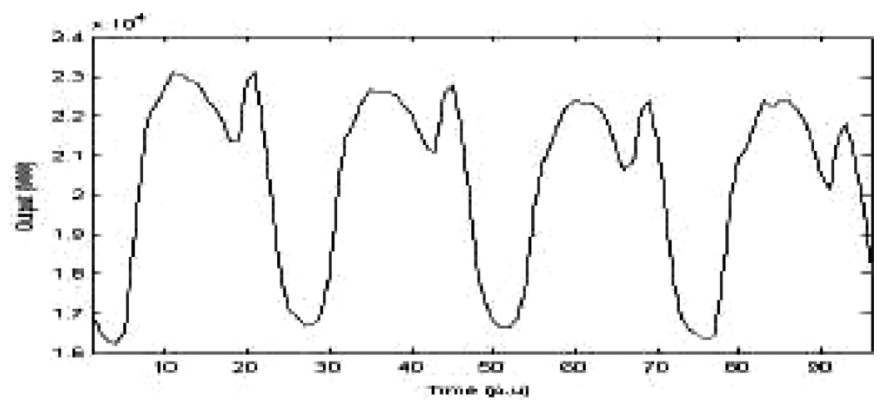

Fig. 4. Unconstrained scheduled quantity.

The system demand varies across hours of a day. Over different weekdays, there is some notable periodicity. For example, demand functions in the same specified hour of different weekdays are almost the same. Supposing the period is $T$ (usually, $T$ is $24 \mathrm{~h}$ ), we have

$$
\left\{\begin{array}{l}
e_{p}(t)=e_{p}(t+T) \\
f_{p}(t)=f_{p}(t+T) .
\end{array}\right.
$$

Because of the non-storability of power energy, the instantaneous market balance requires $D_{d}(t)=\sum_{i=1}^{n} q_{i}(t)$.

Many techniques can be used to model market dynamics in electricity markets, such as ARIMA and linear regression. In our paper, we propose a linear dynamic feedback system, which is based on an individual generator's profit-maximizing behavior.

It is known that an individual generator's profit function is given as the revenue minus the cost, i.e.,

$$
\pi_{i}(t)=p(t) q_{i}(t)-C_{i}\left(q_{i}(t)\right)
$$

where $C_{i}\left(q_{i}\right)=a_{i}+b_{i} q_{i}+(1 / 2) c_{i} q_{i}^{2}$ is generator $i$ 's quadratic production cost function.

From the formulation (3), the first-order condition for optimality should be satisfied to achieve the maximum profit, i.e.,

$$
\frac{\partial \pi_{i}(t)}{\partial q_{i}(t)}=0 .
$$

With the nature of system complexity and variation, it is not expected that generator $i$ can find the optimal $q_{i}(t)$ that satisfies the condition (4) in one shot. Actually, the repeated bidding process of electricity markets provides a mechanism for generator $i$ to refine its generation bid with the revealed system information. That is to say, given the learned system information, generator $i$ can adjust its generation bid in a way to satisfy the condition 
(4). Such models of market dynamics are common in the economic literature [15, Ch. 16, pp. 287-288]). Thus, in a continuous time domain, electricity market dynamics are modeled as $(i=1, \ldots, n)$

$$
\begin{aligned}
\dot{q}_{i}(t) & =\beta_{i}(t) \frac{\partial \pi_{i}(t)}{\partial q_{i}(t)} \\
& =\beta_{i}(t)\left(p(t)+\frac{\partial p(t)}{\partial q_{i}(t)} q_{i}(t)-\left(b_{i}+c_{i} q_{i}(t)\right)\right)
\end{aligned}
$$

where $\beta_{i}(t)$ is a factor representing the speed of adjustment or the preference of adjustment intensity of generator $i$. Equation (5) also indicates that generator $i$ will adjust its generation in the direction of increasing profits. Thus, a dynamic feedback system is obtained where the MCP $p(t)$ is taken as system output feedback. If generator $i$ has a good knowledge of the system inverse demand function (1), the above output feedback system (5) can be easily changed to a state-feedback system, i.e.,

$$
\begin{aligned}
\dot{q}_{i}(t)=A_{i 1}(t) q_{1}(t)+A_{i 2}(t) & q_{2}(t) \\
& +\ldots+A_{\text {in }}(t) q_{n}(t)+q_{i c}(t)
\end{aligned}
$$

where

$A_{\mathrm{ii}}(t)=\beta_{i}(t)\left(-f_{p}(t)-c_{i}+(\partial p(t)) /\left(\partial q_{i}(t)\right)\right), A_{i j}(t)=$ $-\left.\beta_{i}(t) f_{p}(t)\right|_{j \neq i}$. and $q_{i c}(t)=\beta_{i}(t)\left(e_{p}(t)-b_{i}\right)$ can be viewed as an exogenous variable. Because of the periodic property of system demand, parameters in (6) will also be periodic with the same period $(T)$. Note that the adjustment process (6) is a linear state-feedback rule. We can estimate its parameters (including $\beta$ ) via linear regression from historical data.

An example of the above adjustment process is the simple dynamic Cournot competition model. Cournot competition strategy has been well studied in the economic literature [15], and its application in generator bidding is also reported in [16]. The Cournot model means that one generator makes its decision with the belief that others' outputs fixed as the previous, i.e., we have $\left(\partial q_{j}(t)\right) /\left(\partial q_{i}(t)\right)=0(j \neq i)$ and $(\partial p(t)) /\left(\partial q_{i}(t)\right)=-f_{p}(t)$, and $A_{\mathrm{ii}}(t)=\beta_{i}(t)\left(-2 f_{p}(t)-c_{i}\right)$.

As generator bidding in real-time electricity markets is often hourly based, it is better to have a formulation in discrete time domain. Thus, we formulate a corresponding discrete version of (6), i.e.,

$$
\begin{aligned}
q_{i}[t]=A_{i 1}[t-1] q_{1}[t-1] \\
+\ldots+A_{\text {in }}[t-1] q_{n}[t-1]+q_{i c}[t-1] .
\end{aligned}
$$

In matrix form, a linear periodic system yields

$$
\begin{array}{r}
\left(\begin{array}{c}
q_{1}[t] \\
q_{2}[t] \\
\vdots \\
q_{n}[t]
\end{array}\right)=\left(\begin{array}{c}
A_{11}[t-1] A_{12}[t-1] \cdots A_{1 n}[t-1] \\
A_{21}[t-1] A_{22}[t-1] \cdots A_{2 n}[t-1] \\
\vdots \cdot \vdots \\
A_{n 1}[t-1] A_{n 2}[t-1] \cdots A_{\mathrm{nn}}[t-1]
\end{array}\right) \\
\times\left(\begin{array}{c}
q_{1}[t-1] \\
q_{2}[t-1] \\
\vdots \\
q_{n}[t-1]
\end{array}\right)+\left(\begin{array}{c}
q_{1 c}[t-1] \\
q_{2 c}[t-1] \\
\vdots \\
q_{\mathrm{nc}}[t-1]
\end{array}\right) .
\end{array}
$$

If generators adopt the above simple dynamic Cournot strategy, system parameters can be obtained as aforementioned. For other strategic models, the corresponding parameters of the formulation (8) can also be obtained.

Note that with the above dynamic model, the individual generator is making a (dynamic) one-step optimization or single-period optimization.

\section{B. Optimal Control Problem}

As mentioned above, it is more desirable to study long-term optimization considering system dynamics. In this section, an optimal control problem is formulated to investigate individual generator's long-term/multiperiod generation decision behavior.

Without loss of generality, we use generator 1 for illustration (for other generators, the formulation is similar). Now assume that generator 1 undertakes long-term optimization, and its objective is to maximize its aggregated profits over a planning time horizon $N_{T}$

$$
\begin{aligned}
& \max _{\left\{q_{1}[t]\right\}} J_{1}=\max _{\left\{q_{1}[t]\right\}} \\
& \quad \times \sum_{t=1}^{N_{T}}\left(p[t] q_{1}[t]-\left(a_{1}+b_{1} q_{1}[t]+\frac{1}{2} c_{1} q_{1}[t]^{2}\right)\right) .
\end{aligned}
$$

This objective function is subjected to its observation/estimate of rivals' dynamic behavior

$$
\begin{aligned}
& Q_{-1}[t]=A_{-1}[t-1] Q_{-1}[t-1] \\
& +Q_{-1 c}[t-1]+B_{-1}[t-1] q_{1}[t-1]
\end{aligned}
$$

where $Q_{-1}[t]$ is the generation vector from generator 1's rivals, $Q_{-1}[t]=\left(q_{2}[t], q_{3}[t], \ldots, q_{n}[t]\right)^{T} ; A_{-1}[t-1]$ is the matrix composed by estimated elements $A_{k j}[t](k \neq 1, j \neq$ $1) ; B_{-1}[t-1]$ is the vector of estimated elements $A_{k 1}[t](k \neq$ 1 ), and $Q_{-1 c}[t]$ is the vector of exogenous variable from generator 1's rivals, $Q_{-1 c}[t]=\left(q_{2 c}[t], q_{3 c}[t], \ldots, q_{\mathrm{nc}}[t]\right)^{T}$. The observed/estimated $A_{-1}[t-1], B_{-1}[t-1]$ and $Q_{-1 c}[t-1]$ are also periodic with a period $T$.

Equation (10) is also known as the system (estimated) state equation in an optimal control formulation.

The system output equation is the determination of MCP with (1), rewritten as

$$
p[t]=C[t] Q_{-1}[t]+D[t] q_{1}[t]+e_{p}[t]
$$

where $C[t]=\left(-f_{p}[t],-f_{p}[t], \cdots,-f_{p}[t]\right)$ and $D[t]=-f_{p}[t]$. Later on, (11) is substituted into the objective function (9) to solve for a state-feedback control rule.

\section{System STABility AND OPTIMAL STATE-FEEDBACK CONTROL}

In this section, the issue of system stability and the properties of optimal control system are discussed, and an optimal statefeedback control rule is derived via a sweeping method. 


\section{A. Stability of Linear Periodic System}

From the Floquet theory, it is well known that a linear periodic system can be transformed into an equivalent linear timeinvariant system by some Lyapunov transformation, and at the same time, the system stability property is preserved [17]. For our discrete linear periodic system (10), such a transformation can always be done with a "lifting method" [18].

First, define the system monodromy matrix as

$$
\Psi[T]=A_{-1}[T-1] A_{-1}[T-2] \ldots A_{-1}[0]
$$

Concerning system stability issues, we have the following lemmas. (The proof of Lemma 1 and Lemma 4 can be found in [18], and proofs of Lemma 2 and Lemma 3 can be found in Appendix A.)

Lemma 1: The open-loop system (10) is stable if and only if all eigenvalues of matrix $\Psi[T]$ belong to the open unit disk.

Lemma 2: A sufficient condition for all eigenvalues of matrix $\Psi[T]$ to belong to the open unit disk is that all eigenvalues of matrix $A_{-1}[t]$ in one period $T$ belong to the open unit disk.

Lemma 3: For a stable linear periodic system (10), the system stable solution is periodic.

\section{B. Properties of Optimal Control System}

Lemma 4: For a discrete linear periodic system (10), if it is stabilizable and detectable, then there is the unique optimal control rule (from the discrete periodic Riccati equation), and the closed-loop system is stable. Furthermore, the stable solution is also periodic.

Usually, if one system is stable (in the sense of bounded input and bounded output), then it is stabilizable. Our studied optimal control system for generation decisions is always stabilizable (under the condition given by Lemmas 1 or 2) and detectable; therefore, the unique optimal control rule can always been found. The obtained closed-loop system is also stable, which implies that the unique market equilibrium is guaranteed.

Note that these lemmas for system stability are general. Even if there are multiple generators who all use the optimal control approach, these lemmas still give the conditions for system stability.

\section{State-Feedback Control}

To be robust against system uncertainties, the optimal control rule should depend on system information, such as system state variables. Therefore, the state-feedback control rule is investigated. For solving the developed optimal control problem, a Hamilton function is formulated, given as

$$
\begin{aligned}
H[t]= & \left(p[t] q_{1}[t]-\left(a_{1}+b_{1} q_{1}[t]+\frac{1}{2} c_{1} q_{1}[t]^{2}\right)\right) \\
& +\lambda[t+1]^{T}\left(A_{-1}[t] Q_{-1}[t]+B_{-1}[t] q_{1}[t]+Q_{-1 c}[t]\right) \\
= & \left(C[t] Q_{-1}[t]+D[t] q_{1}[t]+e_{p}[t]\right) q_{1}[t] \\
& -\left(a_{1}+b_{1} q_{1}[t]+\frac{1}{2} c_{1} q_{1}[t]^{2}\right) \\
+ & \lambda[t+1]^{T}\left(A_{-1}[t] Q_{-1}[t]+B_{-1}[t] q_{1}[t]+Q_{-1 c}[t]\right) .
\end{aligned}
$$

Note that the last term in (13) considers the impacts of current decisions on future profits.

The Pontryagin maximum principle gives the necessary conditions for optimality (first-order condition)[19]

$$
\begin{aligned}
& \lambda[t]=\frac{\partial H[t]}{\partial Q_{-1}[t]}=C[t]^{T} q_{1}[t]+A_{-1}[t]^{T} \lambda[t+1] \\
& \frac{\partial H[t]}{\partial q_{1}[t]} 0 \Rightarrow\left(C[t] Q_{-1}[t]+D[t] q_{1}[t]+e_{p}[t]\right)+D[t] q_{1}[t] \\
&-\left(b_{1}+c_{1} q_{1}[t]\right)+B_{-1}[t]^{T} \lambda[t+1]=0 \\
& \Rightarrow q_{1}[t] \frac{B_{-1}[t]^{T} \lambda[t+1]-b_{1}+e_{p}[t]+C[t] Q_{-1}[t]}{c_{1}-2 D[t]} \\
& Q_{-1}[t+1]= \frac{\partial H[t]}{\partial q_{1}[t]}=A_{-1}[t] Q_{-1}[t]+Q_{-1}[t]+B_{-1}[t] q_{1}[t] .
\end{aligned}
$$

The sufficient condition for a maximum (second-order condition) is

$$
\frac{\partial^{2} H[t]}{\partial^{2} q_{1}[t]}=2 D[t]-c_{1}=-2 f_{p}[t]-c_{1}<0 .
$$

The condition (17) is always satisfied. This property is due to the continuity and concavity of the objective function (9).

The above optimal control problem does not belong to the standard linear-quadratic regulator (LQR) class, as its objective function multiplies state and control variables (the term $\left.C[t] Q_{-1}[t] q_{1}[t]\right)$. However, the common sweeping method for solving the standard LQR problem is still applicable, as shown in [20]. To solve above specific two-point boundary (14)-(16), we propose a sweeping method (details in Appendix B) and obtain the optimal state-feedback control rule, shown in (18) at the bottom of the page, where both $S[t]$ and $M[t]$ are known

$$
\begin{aligned}
q_{1}[t]= & \frac{B_{-1}[t]^{T} S[t+1] A_{-1}[t]+C[t]}{c_{1}-2 D[t]-B_{-1}[t]^{T} S[t+1] B_{-1}[t]} \\
& \times\left\{A_{-1}[t-1] Q_{-1}[t-1]+B_{-1}[t-1] q_{1}[t-1]+Q_{-1 c}[t-1]\right\} \\
& +\frac{B_{-1}[t]^{T} S[t+1] Q_{-1 c}[t]+B_{-1}[t]^{T} M[t+1]-b_{1}+e_{p}[t]}{c_{1}-2 D[t]-B_{-1}[t]^{T} S[t+1] B_{-1}[t]}
\end{aligned}
$$


as Riccati-like equations, shown in (19) and (20) at the bottom of the page, where terminal conditions are $S\left[N_{T}+1\right]=0$ and $M\left[N_{T}+1\right]=0$. As Lemma $1 \sim 4$ guarantee the existence of an unique optimal control rule and system stability, the state-feedback control rules (18)-(20) will reach their steady states, and system convergence is also assured.

Note that the state-feedback gain of control rule (18) is optimized with respect to system parameters. However, the statefeedback gain of adjustment process (7) is derived from a onestep optimization technique (4), and it is not optimized with respect to system parameters.

\section{NUMERICAL EXAMPLES}

Numerical results are given in this section to show the performance of unilateral optimal control for generation decisions. A sensitivity analysis is also given to identify these market factors that affect the performance of optimal control.

\section{A. Production Cost Function and Demand Function}

The production cost function of a generator is given as

$$
C(q)=10+1.5 q+\frac{1}{2} 0.001 q^{2} .
$$

Real load data in the California electricity market [21] are used for the simulation. Figs. 3 and 4 show its unconstrained demand data on 16-20 April 1998.

Figs. 3 and 4 indicate that system demands have notable periodicity. Although there is also some daily demand variation, here for illustration only, one periodic demand function is estimated via linear regression. The values of system demand function parameter $\left(a_{d}, b_{d}\right)$ in a period are calculated as $a_{d}=\{36689,42909,46535,45699,40674,33125,36803$, 39367, 40452, 41704, 42249, 42309, 42430, 42633, 42460, 42136, 40625, 39490, 39944, 42260, 42928, 41167,37473, $37643\} ; b_{d}=\{1062,1781,2204,2114,1537,621,647,685$, $709,723,704,687,705,719,731,749,734,721,735,724,733$, $747,672,1098\}$. The values of the inverse demand function parameters $\left(e_{p}, f_{p}\right)$ can be easily calculated as

$$
e_{p}(t)=\frac{a_{d}(t)}{b_{d}(t)} \text { and } f_{p}(t)=\frac{1}{b_{d}(t)}
$$

\section{B. Results From Single-Period Optimization}

To compare the performance between multiperiod/long-term optimization and single-period/short-term optimization, let us first focus on a benchmark system and study the performance of short-term/single-period optimization. Assume that there are two symmetrical generators [with the same production cost function (21)]. They both undertake the (dynamic) single-period optimization, and their strategic choices are given as (i.e., a simple dynamic Cournot strategy, and the derivation of such a dynamic model is mentioned in Section IV-2)

$$
\begin{aligned}
q_{i}[t]=(1- & \left.\beta_{i}\left(2 f_{p}[t-1]+c_{i}\right)\right) q_{i}[t-1] \\
& -\beta_{i} f_{p}[t-1] q_{j}[t-1]+\beta_{i}\left(e_{p}[t-1]-b_{i}\right)
\end{aligned}
$$

where $(i=1, j=2)$ or $(i=2, j=1)$. With initial conditions $\left[q_{1}(0)=8711(\mathrm{MWh}), q_{2}(0)=8711(\mathrm{MWh})\right]$, parameters $\beta_{i}=60(i=1,2)$, cost function (21), and the above demand functions (22) are used in forward iteration to obtain generators' outputs and the MCPs. With the formulation (3), generator 1's aggregated profits in one steady period $(24 \mathrm{~h})$ is given as $J_{1}^{\text {Cournot }}=(\$) 4388100$. Due to system symmetry, we have $J_{2}^{\text {Cournot }}=(\$) 4388100$.

\section{Performance of Optimal Control}

We assume the same two symmetric generators. Generator 2 still makes (dynamic) single-period optimization, and its dynamic behavior is given in (22) with the above given parameters. Meanwhile, generator 1 undertakes multiperiod generation optimization with a planning time horizon of $216 \mathrm{~h}$. It is also assumed that generator 1 has a good estimate of generator 2's dynamic behavior. Equations (18)-(20) are used to calculate the optimal control rule (i.e., generator 1's output), and (22) is used to calculate generator 2's output. Fig. 5 shows the generators' output trajectories. It is clear that after a short transient time, the steady periodic solution is repeated.

With the above generation outputs and inverse demand function (1), the MCPs are calculated, and with the formulation (3), the generators' profits are also obtained. It is found that in this situation, generator 1's aggregated profit in a steady period is given as $J_{1}^{\mathrm{OC}}=(\$) 4552300$. The profit increment of generator

$$
\begin{aligned}
S[t]= & C[t]^{T} \frac{C[t]}{c_{1}-2 D[t]}+\left(A[t]^{T}+\frac{C[t]^{T} B_{-1}[t]^{T}}{c_{1}-2 D[t]}\right) \\
& \times S[t+1]\left(\left(c_{1}-2 D[t]\right)-B_{-1}[t] B_{-1}[t]^{T} S[t+1]\right)^{-1} \\
& \times\left\{\left(c_{1}-2 D[t]\right) A_{-1}[t]+B_{-1}[t] C[t]\right\} \\
M[t]= & C[t]^{T} \frac{e_{p}[t]-b_{1}}{c_{1}-2 D[t]}+\left(A_{-1}[t]^{T}+\frac{C[t]^{T} B_{-1}[t]^{T}}{c_{1}-2 D[t]}\right) \\
& \times\left(\begin{array}{c}
S[t+1]\left(\left(c_{1}-2 D[t]\right)-B_{-1}[t] B_{-1}[t]^{T} S[t+1]\right)^{-1} \\
\times\left\{B_{-1}[t]\left(B_{-1}[t]^{T} M[t+1]-b_{1}+e_{p}[t]\right)+\left(c_{1}-2 D[t]\right) Q_{-1 c}[t]\right\} \\
\quad+M[t+1]
\end{array}\right)
\end{aligned}
$$




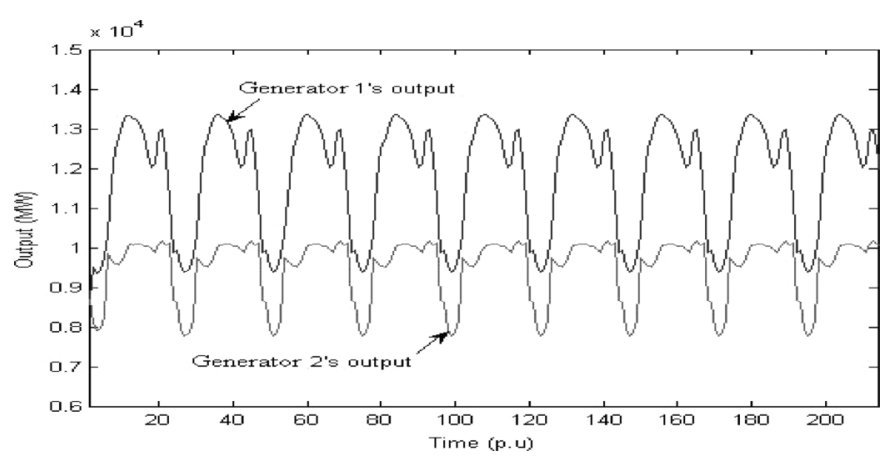

Fig. 5. Trajectory of generators' outputs: Generator 1-optimal control. Generator 2-single-period optimization.

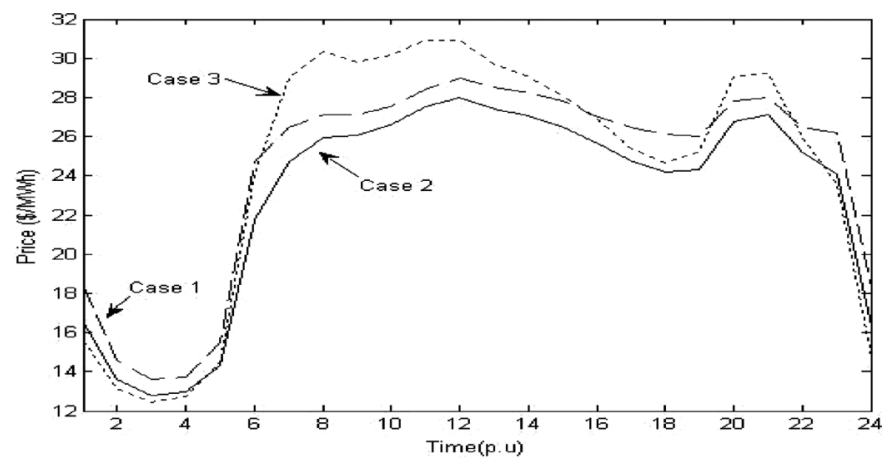

Fig. 6. MCP trajectories in three cases. Case 1-both with strategy (22). Case 2 - one with strategy (22), another with optimal control. Case 3-static Cournot equilibrium price.

1 is $\Delta J_{1}=J_{1}^{\mathrm{OC}}-J_{1}^{\text {Cournot }}=(\$) 164200$, and the percent increase is $3.74 \%$. Meanwhile, generator 2's aggregated profit in one steady period is reduced to $J_{2}^{\text {Cournot }}=(\$) 3838100$.

Furthermore, the completely static Cournot competition outcome can also be obtained with the above data [15]. With the completely static Cournot model, generator 1's aggregated profit in a steady period is given as $J_{1}^{S, C}=(\$) 4411018$.

From the above simulations result, it is known that generator 1 , who makes multiperiod optimization with optimal control, can obtain more profits. This is because generator 1 using optimal control will generate more than the withholding level of other strategies. To have a better view of this fact, let us look at the MCPs in these situations, as shown in Fig. 6.

It is known that for most of the time, MCPs in case 2 are smaller than in cases 1 and 3 . This fact implies that when some generator deviates from the single-period optimization strategy to the "advanced" strategy (such as optimal control), MCPs become smaller. Because of the linear relationship between price and quantity (i.e., $p=e-f D$ ), it is known that the equilibrium quantities in cases 1 and 3 are usually less than those in case 2 . That is to say, an individual generator's "advanced" strategic behavior will have a positive effect on market efficiency and will also benefit consumers. This is similar to what occurs in a static Stackelberg competition game [22].

Because the explicit state-feedback control rule is given by (18)-(20), the above computation is very efficient and done within a few seconds.

\section{Sensitivity Analysis}

It can be expected that system parameters, such as demand function coefficients $\left(a_{d}, b_{d}\right)$, production cost function parameter (e.g., $c_{i}$ ), the adjustment factor $\beta$, and number of market generators $n$, will influence the performance of multiperiod optimization with optimal control. A sensitivity analysis is thus given to show the effect of system parameters' variations. In the simulation, it is assumed that generator 1 will adopt the optimal control, and others follow the single-period optimization strategy. To show the sensitivity of one considered parameter, we change this parameter while keeping the others unchanged. Only the percent profit increase (i.e., $\left.\left(J_{1}^{\mathrm{OC}}-J_{1}^{\text {Cournot }}\right) /\left(J_{1}^{\text {Cournot }}\right) \times 100 \%\right)$ is shown here. The simulation procedure is similar to the one in the above section. Fig. 7 shows the percent profit increase with respect to several system parameters. In the above simulation, the computation is also efficient, and the convergence is fast.

The percent profit increase is one index to measure the performance of long-term/multiperiod generation optimization for the leader firm with optimal control. Generally, if it is rather small, the performance of long-term/multiperiod generation optimization is unsatisfactory; otherwise, the performance is good. According to this criterion and from the above simulation results, the following can be concluded.

1) With the marginal cost function slope increasing, the performance of optimal control will deteriorate, i.e., the more expensive the generation production, the less beneficial the optimal control.

2) With the demand function slope increasing, the performance of optimal control will deteriorate, which means that the more elastic the demand, the less beneficial the optimal control.

3) With the demand function intercept scaling up, the better the performance of optimal control, which implies that the more system demand, the more beneficial to apply optimal control.

4) The more generators in the market using the simple (singleperiod optimization) strategy, the better the performance of optimal control.

5) If rivals' responses (a small $\beta$ ) are slower, the performance of optimal control improves.

\section{DISCUSSION}

\section{A. Consideration of Operating Constraints and Network Constraints}

The current study does not consider many system operational constraints, such as generation capacity limits and ramping up/down limits as well as network constraints. In particular, when network constraints are considered, a key issue is how the system operator prices them and how generators can change those prices [23]. Actually, these constraints will affect both system state variables and control variables. Such issues should be incorporated into our future formulation. Generally, there are two ways to include these constraints [17]. The first alternative is to augment the system Hamilton function with penalty functions that attribute high costs to deviations from the constraints. The second alternative is to use Lagrange 


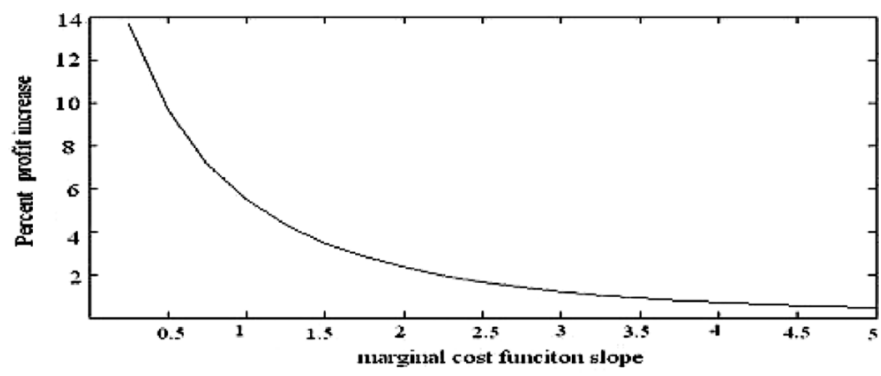

(a)

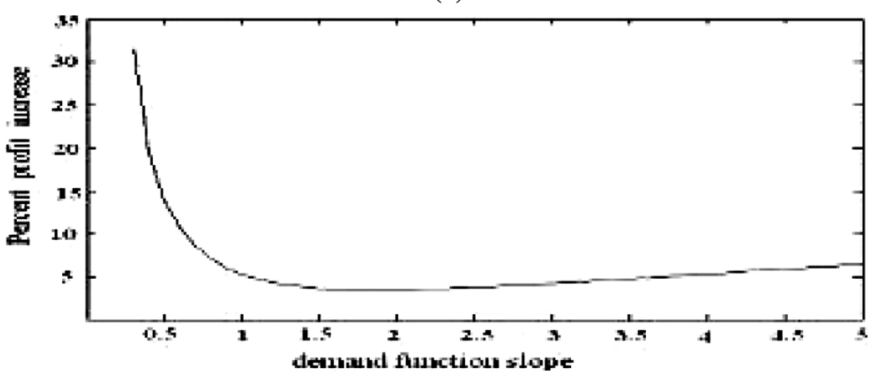

(b)

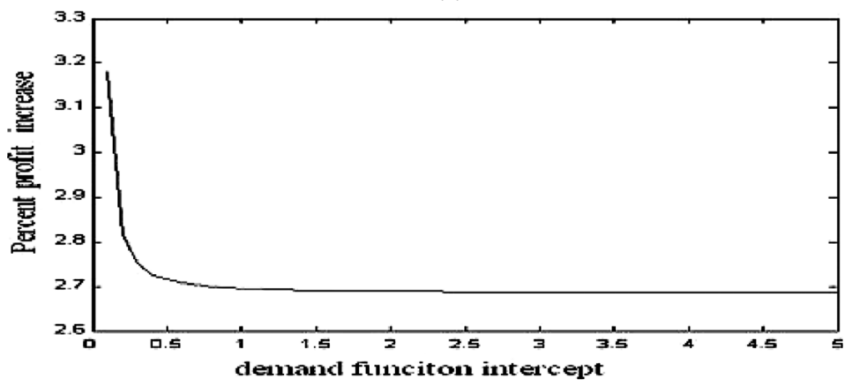

(c)

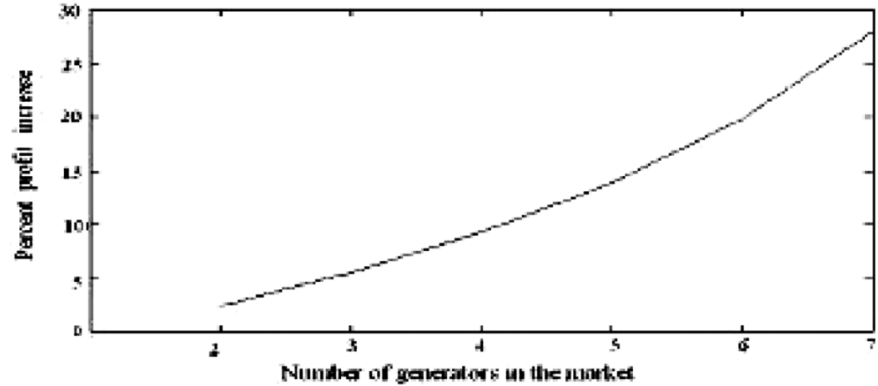

(d)

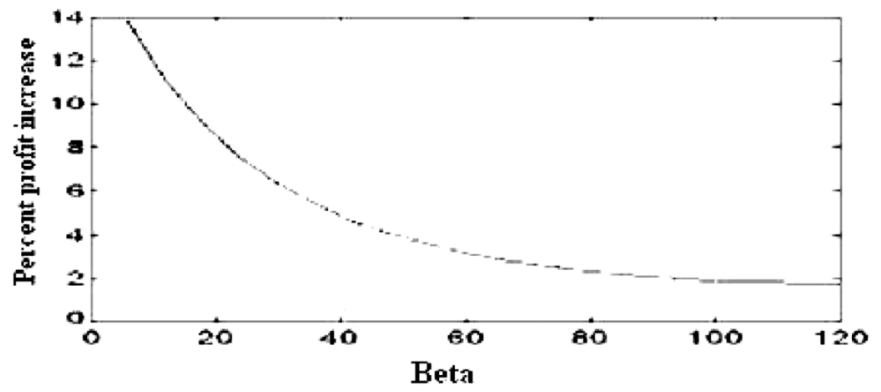

(e)

Fig. 7. Percent profit increase with respect to (a) $c_{i}$; (b) $b_{d}$; (c) $a_{d}$; (d) $n$; and (e) $\beta$.

multipliers to adjoin these constraints to the objective function. To avoid the problem of penalty function selection, the second alternative is suggested. Thus, after including these constraints in our formulation, we obtain a corresponding constrained optimal control problem. To solve it, an augmented system
Hamilton function is then formulated. Necessary conditions for optimality are still given by the Pontryagin maximum principle. Based on these conditions, the optimal solution can be obtained in principle via a similar procedure, as shown in this paper. Of course it should be pointed out that in some situations, the explicit solution may be difficult to obtain, and we should resort to numerical techniques [24].

\section{B. Other Extensions of the Proposed Method}

Although the above formulation considers periodic system demand, it is also applicable to other situations. For instance, if multiple system demand profiles (not necessary periodic) are given along the time horizon, the price-quantity pairs (the generation bid curves) for each time slot can also be generated by the proposed method.

In reality, there are many market uncertainties associated with system demand as well as the observation/estimate of rival behavior; therefore, a stochastic formulation is more preferred. If system uncertainties can be modeled by some known probabilistic process, such as the Gaussian distribution, a corresponding stochastic optimal control (LQG) problem can be formulated. As system stochastic behaviors between different time slots are usually interdependent (e.g., system hourly demands are strongly correlated), it is obvious that we cannot replace system random variables with their expected values and deal with the stochastic system as a deterministic one when solving it (i.e., the certainty equivalent principle [9] does not hold). The obtained system performance would be suboptimal. To obtain the global optimum in this situation, the covariance of system random variables should be incorporated into system formulation.

It is also interesting to explore the situation in which two or more generators apply the optimal control for generation decisions and then study its effect on generators' profits and market efficiency. For these investigations, we conjecture that there will be a kind of "Prisoner Dilemma" [22] (i.e., if more generators are the optimal control type, actually generators' payoffs will decrease). These issues are left for future work.

\section{CONCLUSION}

In this paper, optimal control is applied to study generator bidding in an oligopolistic electricity market. Based on the generators' dynamic profit-maximizing behavior, we model the repeated bidding process in (hourly-based) real-time electricity markets as a dynamic feedback system.

We then formulate an optimal control problem to explore an individual generator's long-term/multiperiod optimization behavior. In our formulation, the periodic property of the system demand is considered.

Several lemmas are included for concerning system stability. Necessary conditions for solving the proposed optimal control problem are given by the Pontryagin maximum principle. Based on these necessary conditions and with a sweeping method, we obtain an optimal state-feedback control rule via backward induction. Numerical results suggest the generator who uses the optimal control can obtain more profits. A sensitivity analysis is also performed afterward, identifying these market factors that affect the performance of optimal control. Future work is 
in progress on extending the proposed method to a general situation with various market uncertainties.

\section{APPENDIX A}

STABILITY OF A LINEAR PERIODIC SYSTEM

Consider a linear periodic system

$$
\begin{aligned}
& Q_{-1}[t]=A_{-1}[t-1] Q_{-1}[t-1] \\
& +Q_{-1 c}[t-1]+B_{-1}[t-1] q_{1}[t-1]
\end{aligned}
$$

where $A_{-1}[t-1]$ and $B_{-1}[t-1]$ are periodic; $Q_{-1 c}[t-1]$ is the state vector, bounded and periodic; and $q_{1}[t-1]$ is the control variable, also bounded. The period is $T$.

From linear system theory, it is known that that system solution is given as

$$
\begin{aligned}
& Q_{-1}[t]=\Phi[t, 0] Q_{-1}[0] \\
& \quad+\sum_{i=0}^{t-1} \Phi[t, i+1]\left(Q_{-1 c}[i]+B_{-1}[i] q_{1}[i]\right)
\end{aligned}
$$

where $\Phi[t, 0]$ is the state transition matrix, defined as

$$
\Phi\left[t, t_{0}\right]=A_{-1}[t-1] A_{-1}[t-2] \ldots A_{-1}\left[t_{0}\right] .
$$

Because of the periodicity of matrix $A_{-1}[t]$, the state transition matrix enjoys the property

$$
\Phi\left[t+T, t_{0}+T\right]=\Phi\left[t, t_{0}\right] .
$$

Define the system monodromy matrix

$$
\Psi[T]=\Phi[T, 0]=A_{-1}[T-1] A_{-1}[T-2] \ldots A_{-1}[0] .
$$

Equation (A2) can be rewritten as shown in (A6) at the bottom of the page, where $\operatorname{res}(\cdot)$ is to obtain the residual, and $\bmod (\cdot)$ is to obtain the module. Such a technique for transforming a linear periodic (time-varying) system to an equivalent linear time-invariant system in the control literature is known as the lifting method [18].

Lemma 2: A sufficient condition for all eigenvalues of matrix $\Psi[T]$ to belong to the open unit disk is that all eigenvalues of matrix $A_{-1}[t]$ in one period belong to the open unit disk.
Proof: If all eigenvalues of normal matrix $A_{-1}[t]$ in one period belong to the open unit disk, we have $\|\Psi[T]\|=$ $A_{-1}[T-1] A_{-1}[T-2] \ldots A_{-1}[0] \|<1$; that is to say that all eigenvalues of matrix $\Psi[T]$ belong to the open unit disk.

Lemma 3: For a stable linear periodic system (A-1), the system stable solution is periodic.

Proof: With (A-6), system state solution $Q_{-1}[t+T]$ is given as

$$
\begin{aligned}
Q_{-1}[t+T]= & \Phi[\operatorname{res}(t, T), 0] \Psi[T]^{\bmod (t, T)+1} Q_{-1}[0] \\
+ & \sum_{i=0}^{\bmod (t-1, T)+1} \Psi[T]^{i} \\
& \times \sum_{j=0}^{T-1} \Phi[j, 0]\left(Q_{-1 c}[j]+B_{-1}[j] q_{1}[j]\right) \\
& +\sum_{j=0}^{\operatorname{res}(t+T-1, T)} \Phi[j, 0]\left(Q_{-1 c}[j]+B_{-1}[j] q_{1}[j]\right) .
\end{aligned}
$$

As the system is stable, we have $\Psi[T]^{\bmod (t, T)} \rightarrow 0(t \rightarrow \infty)$ and

$$
\begin{aligned}
Q_{-1}[t+T]= & \Phi[\operatorname{res}(t, T), 0] \Psi[T]^{\bmod (t, T)+1} Q_{-1}[0] \\
& +\sum_{i=0}^{\bmod (t-1, T)+1} \Psi[T]^{i} \\
& \times \sum_{j=0}^{T-1} \Phi[j, 0]\left(Q_{-1 c}[j]+B_{-1}[j] q_{1}[j]\right) \\
& +\sum_{j=0}^{\operatorname{res}(t+T-1, T)} \Phi[j, 0]\left(Q_{-1 c}[j]+B_{-1}[j] q_{1}[j]\right) \\
\bmod (t-1, T)+1 & \sum_{i=0} \Psi[T]^{i} \sum_{j=0}^{T-1} \Phi[j, 0]\left(Q_{-1 c}[j]+B_{-1}[j] q_{1}[j]\right) \\
& +\sum_{j=0}^{\operatorname{res}(t+T-1, T)} \Phi[j, 0]\left(Q_{-1 c}[j]+B_{-1}[j] q_{1}[j]\right) \\
= & \sum_{i=0}^{\bmod (t-1, T)} \Psi[T]^{i} \sum_{j=0}^{T-1} \Phi[j, 0]\left(Q_{-1 c}[j]+B_{-1}[j] q_{1}[j]\right) \\
& +\sum_{j=0}^{\operatorname{res}(t+T-1, T)} \Phi[j, 0]\left(Q_{-1 c}[j]+B_{-1}[j] q_{1}[j]\right) \\
= & Q_{-1}[t] .
\end{aligned}
$$

$$
\begin{aligned}
Q_{-1}[t]= & \Phi[\operatorname{res}(t, T), 0] \Psi[T]^{\bmod (t, T)} Q_{-1}[0] \\
& +\sum_{i=0}^{t-1}\left(\begin{array}{r}
\Phi[\operatorname{res}(t-i-1, T), 0] \Psi[T] \bmod (t-1-i, T) \\
\times\left(Q_{-1 c}[\operatorname{res}(i, T)]+B_{-1}[\operatorname{res}(i, T)] q_{1}[\operatorname{res}(i, T)]\right)
\end{array}\right) \\
= & \Phi[\operatorname{res}(t, T), 0] \Psi[T]^{\bmod (t, T)} Q_{-1}[0] \\
& +\sum_{i=0}^{\bmod (t-1, T)} \Psi[T]^{i} \sum_{j=0}^{T-1} \Phi[j, 0]\left(Q_{-1 c}[j]+B_{-1}[j] q_{1}[j]\right)+ \\
& +\sum_{j=0}^{\operatorname{res}(t-1, T)} \Phi[j, 0]\left(Q_{-1 c}[j]+B_{-1}[j] q_{1}[j]\right)
\end{aligned}
$$


APPENDIX B

Optimal State-FeEdBACK CONTROL RULE

For the Hamilton function

$$
\begin{aligned}
& H[t]=\left(C[t] Q_{-1}[t]+D[t] q_{1}[t]+e_{p}[t]\right) q_{1}[t] \\
&-\left(a_{1}+b_{1} q_{1}[t]+\frac{1}{2} c_{1} q_{1}[t]^{2}\right) \\
& \lambda[t+1]^{T}\left(A_{-1}[t] Q_{-1}[t]+B_{-1}[t] q_{1}[t]+Q_{-1 c}[t]\right) .
\end{aligned}
$$

The necessary conditions for optimality based on maximum principle are listed as shown in (B2)-(B4) at the bottom of the page. A sweeping method is used to find the state feedback control rule, i.e., assuming there is linear relationship between ad-joint variable $\lambda[t]$ and system state variable

$$
\lambda[t]=S[t] Q_{-1}[t]+M[t],
$$

With (B3)-(B5), this yields (B6), shown on the bottom of the page. Solving for $Q_{-1}[t+1]$ gives (B7), shown at the bottom of the page. From (B5) and (B7), we have (B8), shown at the bottom of the page. With (B5) and (B8), we have (B9), shown at the bottom of the next page. For the condition (B9) to be

$$
\begin{aligned}
\lambda[t]= & \frac{\partial H[t]}{\partial Q_{-1}[t]}=C[t]^{T} q_{1}[t]+A_{-1}[t]^{T} \lambda[t+1] \\
Q_{-1}[t+1]= & \frac{\partial H[t]}{\partial \lambda[t+1]}=A_{-1}[t] Q_{-1}[t]+B_{-1}[t] q_{1}[t]+Q_{-1 c}[t] \\
\frac{\partial H[t]}{\partial q_{1}[t]}= & 0 \Rightarrow\left(C[t] Q_{-1}[t]+D[t] q_{1}[t]+e_{p}[t]\right) \\
& +D[t] q_{1}[t]-\left(b_{1}+c_{1} q_{1}[t]\right)+B_{-1}[t]^{T} \lambda[t+1]=0 \\
\Rightarrow q_{1}[t]= & \frac{B_{-1}[t]^{T} \lambda[t+1]-b_{1}+e_{p}[t]+C[t] Q_{-1}[t]}{c_{1}-2 D[t]}
\end{aligned}
$$

$$
\begin{aligned}
Q_{-1}[t+1]= & A_{-1}[t] Q_{-1}[t]+Q_{-1 c}[t] \\
& +B_{-1}[t] \frac{B_{-1}[t]^{T} \lambda[t+1]-b_{1}+e_{p}[t]+C[t] Q_{-1}[t]}{c_{1}-2 D[t]} \\
= & A_{-1}[t] Q_{-1}[t]+Q_{-1 c}[t] \\
& +B_{-1}[t] \frac{B_{-1}[t]^{T}\left(S[t+1] Q_{-1}[t+1]+M[t+1]\right)-b_{1}+e_{p}[t]+C[t] Q_{-1}[t]}{c_{1}-2 D[t]}
\end{aligned}
$$

$$
\begin{aligned}
Q_{-1}[t+1]=\left(\left(c_{1}-2 D[t]\right)-B_{-1}[t] B_{-1}[t]^{T} S[t+1]\right)^{-1} & \times\left\{\begin{array}{c}
\left(c_{1}-2 D[t]\right) A_{-1}[t] Q_{-1}[t]+\left(c_{1}-2 D[t]\right) Q_{-1 c}[t]+ \\
+B_{-1}[t]\left(B_{-1}[t]^{T} M[t+1]-b_{1}+e_{p}[t]+C[t] Q_{-1}[t]\right)
\end{array}\right\}
\end{aligned}
$$

$$
\begin{aligned}
\lambda[t+1]= & S[t+1] Q_{-1}[t+1]+M[t+1] \\
= & S[t+1]\left(\left(c_{1}-2 D[t]\right)-B_{-1}[t] B_{-1}[t]^{T} S[t+1]\right)^{-1} \\
& \times\left\{\begin{array}{c}
\left(c_{1}-2 D[t]\right) A_{-1}[t] Q_{-1}[t]+\left(c_{1}-2 D[t]\right) Q_{-1 c}[t] \\
\quad+B_{-1}[t]\left(B_{-1}[t]^{T} M[t+1]-b_{1}+e_{p}[t]+C[t] Q_{-1}[t]\right)
\end{array}\right\} \\
& +M[t+1]]
\end{aligned}
$$


satisfied for all state sequences, clearly we must have (B10) and (B11), shown at the bottom of the page, where terminal conditions are $S\left[N_{T}+1\right]=0$ and $M\left[N_{T}+1\right]=0$. The sequence $(S[t], M[t])$ can be calculated with (B10) and (B11) via backward induction. Matrix $S[t]$ and $M[t]$ are also known as Riccati-like equations.
With $(S[t], M[t])$ calculated via (B10) and (B11), (B5) is substituted into (B4) to obtain the optimal control rule, given as shown in (B12) at the bottom of the page. Solving for $q_{1}[t]$ gives (B13), shown at the bottom of the page. At time $t, Q_{-1}[t-1]$ and $q_{1}[t-1]$ are known, and the optimal control $q_{1}[t]$ can be calculated with (B13).

$$
\begin{aligned}
S[t] Q_{-1}[t]+M[t] & \\
= & C[t]^{T} \frac{B_{-1}[t]^{T} \lambda[t+1]-b_{1}+e_{p}[t]+C[t] Q_{-1}[t]}{c_{1}-2 D[t]}+A_{-1}[t]^{T} \lambda[t+1] \\
= & C[t]^{T} \frac{e_{p}[t]+C[t] Q_{-1}[t]-b_{1}}{c_{1}-2 D[t]}+\left(A_{-1}[t]^{T}+\frac{C[t]^{T} B_{-1}[t]^{T}}{c_{1}-2 D[t]}\right) \lambda[t+1] \\
= & C[t]^{T} \frac{e_{p}[t]+C[t] Q_{-1}[t]-b_{1}}{c_{1}-2 D[t]}+\left(A_{-1}[t]^{T}+\frac{C[t]^{T} B_{-1}[t]^{T}}{c_{1}-2 D[t]}\right) \\
& \times\left(\begin{array}{l}
S[t+1]\left(\left(c_{1}-2 D[t]\right)-B_{-1}[t] B_{-1}[t]^{T} S[t+1]\right)^{-1} \\
\times\left\{\begin{array}{c}
\left(c_{1}-2 D[t]\right) A_{-1}[t] Q^{-}[t]+\left(c_{1}-2 D[t]\right) Q_{-1 c}[t] \\
+B_{-1}[t]\left(B_{-1}[t]^{T} M[t+1]-b_{1}+e_{p}[t]+C[t] Q_{-1}[t]\right)
\end{array}\right\}+M[t+1]
\end{array}\right)
\end{aligned}
$$

$$
\begin{aligned}
S[t]= & C[t]^{T} \frac{C[t]}{c_{1}-2 D[t]}+\left(A_{-1}[t]^{T}+\frac{C[t]^{T} B_{-1}[t]^{T}}{c_{1}-2 D[t]}\right) \\
& \times S[t+1]\left(\left(c_{1}-2 D[t]\right)-B_{-1}[t] B_{-1}[t]^{T} S[t+1]\right)^{-1} \\
& \times\left\{\left(c_{1}-2 D[t]\right) A_{-1}[t]+B_{-1}[t] C[t]\right\} \\
M[t]= & C[t]^{T} \frac{e_{p}[t]-b_{1}}{c_{1}-2 D[t]}+\left(A_{-1}[t]^{T}+\frac{C[t]^{T} B_{-1}[t]^{T}}{c_{1}-2 D[t]}\right) \\
& \times\left(\begin{array}{c}
S[t+1]\left(\left(c_{1}-2 D[t]\right)-B_{-1}[t] B_{-1}[t]^{T} S[t+1]\right)^{-1} \\
\quad \times\left\{B_{-1}[t]\left(B_{-1}[t]^{T} M[t+1]-b_{1}+e_{p}[t]\right)+\left(c_{1}-2 D[t]\right) Q_{-1 c}[t]\right\} \\
\quad+M[t+1]
\end{array}\right)
\end{aligned}
$$

$$
\begin{aligned}
q_{1}[t]= & \frac{B_{-1}[t]^{T}\left(S[t+1] Q_{-1}[t+1]+M[t+1]\right)-b_{1}+e_{p}[t]+C[t] Q_{-1}[t]}{c_{1}-2 D[t]} \\
= & \frac{B_{-1}[t]^{T} S[t+1] A_{-1}[t]+C[t]}{c_{1}-2 D[t]} Q_{-1}[t] \\
& +\frac{B_{-1}[t]^{T}\left(S[t+1]\left(B_{-1}[t] q_{1}[t]+Q_{-1 c}[t]\right)+M[t+1]\right)-b_{1}+e_{p}[t]}{c_{1}-2 D[t]}
\end{aligned}
$$

$$
\begin{aligned}
q_{1}[t]= & \frac{B_{-1}[t]^{T} S[t+1] A_{-1}[t]+C[t]}{c_{1}-2 D[t]-B_{-1}[t]^{T} S[t+1] B_{-1}[t]} \\
& \times\left\{A_{-1}[t-1] Q_{-1}[t-1]+B_{-1}[t-1] q_{1}[t-1]+Q_{-1 c}[t-1]\right\} \\
& +\frac{B_{-1}[t]^{T} S[t+1] Q_{-1 c}[t]+B_{-1}[t]^{T} M[t+1]-b_{1}+e_{p}[t]}{c_{1}-2 D[t]-B_{-1}[t]^{T} S[t+1] B_{-1}[t]}
\end{aligned}
$$




\section{REFERENCES}

[1] S. Borenstein and J. Bushnell, "Market power in electricity markets: Beyond concentration measures," Energy J., vol. 20, no. 4, pp. 65-88, 1999.

[2] H. Song, C.-C. Liu, J. Lawarrée, and R. W. Dahlgren, "Optimal electricity supply bidding by Markov decision process," IEEE Trans. Power Syst., vol. 15, no. 2, pp. 618-624, May. 2000.

[3] F. Wen and A. K. David, "Optimal bidding strategies and modeling of imperfect information among competitive generators," IEEE Trans. Power Syst., vol. 16, no. 1, pp. 15-21, Feb. 2001.

[4] X. Guan, Y.-C. Ho, and F. Lai, "An ordinal optimization based bidding strategy for electric power suppliers in the daily energy market," IEEE Trans. Power Syst., vol. 16, no. 4, pp. 788-797, Nov. 2001.

[5] E. Ni, P. B. Luh, and S. Rourke, "Optimal integrated generation bidding and scheduling with risk management under a deregulated power market," IEEE Trans. Power Syst., vol. 19, no. 1, pp. 600-609, Feb. 2004.

[6] H. Song, C.-C. Liu, and J. Lawarrée, "Nash equilibrium bidding strategies in a bilateral electricity market," IEEE Trans. Power Syst., vol. 17, no. 1, pp. 73-79, Feb. 2002.

[7] J. B. Park, B. H. Kim, J. H. Kim, M. H. Jung, and J. K. Park, "A continuous strategy game for power transactions analysis in competitive electricity markets," IEEE Trans. Power Syst., vol. 16, no. 4, pp. 847-855, Nov. 2001.

[8] R. Baldick, R. Grant, and E. Kahn, "Linear supply function equilibrium: Generalizations, applications and limitations," UCEI Working Paper Aug. 2000 [Online]. Available: http://www.ucei.Berkeley.edu/ PDF/pwp078.pdf

[9] F. Alvarado, "The stability of power system markets," IEEE Trans. Power Syst., vol. 14, no. 2, pp. 505-511, May 1999.

[10] P. Visudhiphan and M. D. Ilic, "Dynamic games-based modeling of electricity markets," in Proc. IEEE Power Eng. Soc. Winter Meeting, 1999, vol. 1, pp. 274-281.

[11] A. R. Kian, J. J. B. Cruz, and R. J. Thomas, "Bidding strategies in oligopolistic dynamic electricity double-Sided auctions," IEEE Trans. Power Syst., vol. 20, no. 1, pp. 50-58, Feb. 2005.

[12] Y. F. Liu, Y. X. Ni, and F. F. Wu, "Control theory application in power market stability analysis," in Proc. Electric Utility Deregulation, Restructuring, Power Technologies, Hong Kong, Apr. 2004, vol. 2, pp. $562-569$.

[13] M. Shahidehpour and M. Almoush, Restructured Electrical Power Systems: Operation, Trading, and Volatility. New York: Marcel Dekker, 2001.
[14] E. R. Branch, "Short run income elasticity of demand for residential electricity using consumer expenditure survey data," Energy J., vol. 14, no. 3, pp. 111-121, 1993.

[15] H. R. Varian, Microeconomic Analysis, 3rd ed. : Norton, 1992.

[16] J. Contreras, O. Candiles, J. I. de la Fuente, and T. Gómez, "A cobweb bidding model for competitive electricity markets," IEEE Trans. Power Syst., vol. 17, no. 1, pp. 148-153, Feb. 2002.

[17] C. T. Chen, Linear System Theory and Design, 3rd ed. London, U.K.: Oxford Univ. Press, 1998.

[18] S. Bittanti, A. J. Laub, and J. C. Willems, The Riccati Equation. Berlin, Germany: Springer-Verlag, 1991.

[19] R. F. Stengel, Stochastic Optimal Control: Theory and Application. New York: Wiley, 1986.

[20] W. E. Schimitendorf and S. J. Citron, "On the applicability of the sweep method to optimal control problems," IEEE Trans. Autom. Control, vol. AC-14, no. 1, pp. 69-72, Jan. 1969.

[21] [Online]. Available: http://www.ucei.berkeley.edu/.

[22] D. Fudenberg and J. Tirole, Game theory. Cambridge, MA: MIT Press, 1991

[23] S. S. Oren, T. S. Pablo, P. Varaiya, and F. F. Wu, "Nodal prices and transmission rights: A critical appraisal," Elect. J., vol. 8, no. 3, pp. 24-35, 1995.

[24] K. L. Teo, C. J. Goh, and K. H. Wong, A Unified Computational Approach To Optimal Control Problems. New York: Wiley, 1991.

Youfei Liu (S'04) received the B.S. and M.S. degrees in control theory and control engineering from University of Science and Technology of China, Hefei, China, in 1999 and 2002, respectively. He is currently pursuing the Ph.D. degree in the Electrical and Electronics Engineering Department, Hong Kong University.

His current research interest is the analysis of dynamics and stability of power market.

Felix F. Wu (F'89) received the Ph.D. degree from University of California at Berkeley (UCB).

$\mathrm{He}$ is now a Chair Professor in the Electrical and Electronics Engineering Department, Hong Kong University. Prior to that, he was a Professor and ViceChair of Department of Electrical Engineering and Computer Sciences at UCB. His research interests are electric energy industry restructuring, power system investment planning, design of modern control centers, and distributed processing. 